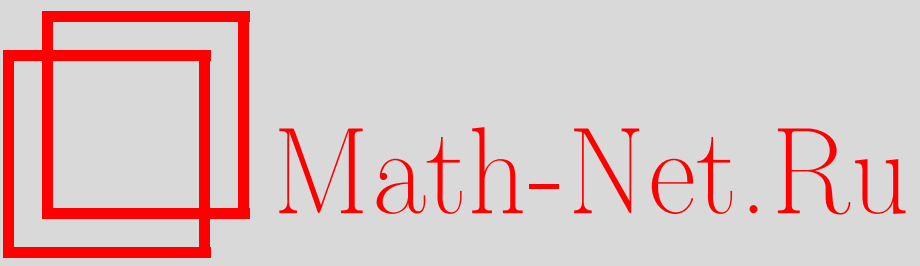

А. Н. Данилов, О касательных кольцах к многообразиям мультиоператорных групп, УМН, 2000, том 55, выпуск 3, 175-176

DOI: https://doi.org/10.4213/rm299

Использование Общероссийского математического портала Math-Net.Ru подразумевает, что вы прочитали и согласны с пользовательским соглашением

http://www . mathnet.ru/rus/agreement

Параметры загрузки:

IP : 3.93 .64 .190

26 апреля 2023 г., 16:29:46 


\section{О КАСАТЕЛЬНЫХ КОЛЬЦАХ К МНОГООБРАЗИЯМ МУЛЬТИОПЕРАТОРНЫХ ГРУПП}

\section{А.Н. ДАнилов}

В данной работе изучаются некоторые свойства касательных колец к модулярным многообразиям, которые мыслятся при этом как основные объекты, возникающие в конструкции общего представления Магнуса, предложенной в [1]. В этой работе все кольца ассоциативны и с единицей, модули унитарны.

Представление Магнуса играет важную роль в теории групп (см. [2] и [3]), ассоциативных алгебр и алгебр Ли (см. [4]). При обобщении на произвольные модулярные многообразия возникает понятие касательного кольца $\mathbf{R}=\mathbf{R}_{V}(\mathbf{A}, p)$ к многообразию $V$ с фиксированным термом Гумма $p$ в алгебре $\mathbf{A}$, см. [1]. Касательное кольцо $\mathbf{R}$ определяется как аддитивная категория, объектами которой являются элементы алгебры $\mathbf{A}$ и которая порождается символами $\frac{\partial f}{\partial i}(\vec{a})$, где $f \in F_{V}(n)$ (свободная алгебра ранга $\left.n\right), \vec{a} \in A^{n}, i \in\{1, \ldots, n\}$, являющимися морфизмами $a_{i} \rightarrow f(\vec{a})$, и задается соотношениями:

$$
\begin{aligned}
& \text { 1) } \frac{\partial(f(\vec{g}))}{\partial i}(\vec{a})=\sum_{j=1}^{m}\left(\frac{\partial f}{\partial j}(\vec{g}(\vec{a}))\right)\left(\frac{\partial g_{j}}{\partial i}(\vec{a})\right) \\
& \text { 2) } \frac{\partial x_{j}}{\partial i}(\vec{a})=\delta_{i j}= \begin{cases}1: a_{i} \rightarrow a_{i}, & i=j, \\
0: a_{i} \rightarrow a_{j}, & i \neq j,\end{cases} \\
& \text { 3) } \frac{\partial p}{\partial 1}(a, a, a)=1 .
\end{aligned}
$$

Пусть $V \ni \mathbf{A} \cong \mathbf{F}_{V}(X) / \theta$, где $\theta \in \operatorname{Con} \mathbf{F}_{V}(X)$ (конгруэнция свободной алгебры), $\theta=\operatorname{ker} \pi$, $\pi: \mathbf{F}_{V}(X) \rightarrow \mathbf{A}$ - естественный эпиморфизм; вместо $\pi(x)$ будем также писать $\bar{x}$. Рассмотрим свободный $\mathbf{R}$-модуль $\mathbf{H}=\{\mathbf{H}(a) \mid a \in A\}$, порожденный множеством $\left\{e_{x} \in H(\bar{x}) \mid x \in X\right\}$. На $H$ вводится структура $V$-алгебры по правилу $f(\vec{h})=\sum_{i=1}^{n}\left(\frac{\partial f}{\partial i}(\vec{a})\right) h_{i}$, где $h_{i} \in H\left(a_{i}\right), f$-основная операция арности $n>0$, и $c=0 \in H(c)$ для каждой нульарной основной операции $c$. Представление $М$ агнуса $\mu$ - это такой гомоморфизм $V$-алгебр $\mathbf{F}_{V}(X) \rightarrow \mathbf{H}$, что $\mu(x)=e_{x}$ на $X$. Если $\operatorname{ker} \mu=[\theta, \theta]$, то представление Магнуса дает описание $\mathbf{F}_{V}(X) /[\theta, \theta]$ в терминах $\mathbf{F}_{V}(X) / \theta$.

В случае, когда $p$ - обратимый мальцевский терм, удается переформулировать определение представления Магнуса в терминах кольца и свободного модуля над ним, см. [1]. Пусть $\mathbf{R}-$ кольцо, порожденное символами $\mathscr{D}_{i} f(\vec{a})$ и заданное соотношениями:

1) $\mathscr{D}_{i}(f(\vec{g}))(\vec{a})=\sum_{j=1}^{m} \mathscr{D}_{j} f(\vec{g}(\vec{a})) \cdot \mathscr{D}_{i} g_{j}(\vec{a})$,

2) $\mathscr{D}_{i} x_{j}(\vec{a})=\delta_{i j}$

3) $\mathscr{D}_{1} p(a, a, a) \stackrel{=}{=}$.

Пусть $\mathbf{U}$ - свободный $\mathbf{R}$-модуль с базой $X$, и пусть множество $H=A \times U$. Тогда на $H$ вводится структура $V$-алгебры по правилу $f(\vec{h})=\left(f(\vec{a}), \sum_{i=1}^{n} \mathscr{D}_{i} f(\vec{a}) u_{i}\right)$, где $h_{i}=\left(a_{i}, u_{i}\right), f-$ основная операция арности $n>0$, и $c=(c, 0)$ для каждой нульарной основной операции $c$. Представление Магнуса $\mu$ - это такой гомоморфизм $V$-алгебр $\mathbf{F}_{V}(X) \rightarrow \mathbf{H}$, что $\mu(x)=(\bar{x}, x)$ на $X$. В этом случае касательным кольцом называют введенное здесь кольцо $\mathbf{R}$, т.е. проводят коррекцию определения касательного кольца. Однако для ряда важных многообразий (а именно, многообразий $\Omega$-групп) касательное кольцо оказывается избыточным даже после этой коррекции. Поэтому предлагается очередная коррекция.

Заметим, что в любом многообразии $\Omega$-групп можно взять $p=x-y+z$. Кроме инфиксной мы будем использовать и префиксную запись групповых операций: $A(x, y)=x+y$ и $I(x)=-x$. Согласно сказанному выше касательное кольо $\mathbf{R}$ к многообразию всех $\Omega$-групп в $\Omega$-группе $\mathbf{G}-$ это некоторое кольцо, порожденное символами $\mathscr{D}_{j} A(a, b), \mathscr{D} I(a)$ и $\mathscr{D}_{i} f(\vec{a})$.

Работа выполнена при частичной поддержке Российского фонда фундаментальных исследований (грант № 99-01-00382). 
Теорема 1. Для любого многообразия $\Omega$-групп $\mathbf{R} \cong \mathbb{Z} \mathbf{F}_{2}(G \backslash\{0\}) * \mathbf{R}^{\prime}$, где через $\mathbf{F}_{2}$ обозначена свободная группа, а кольцо $\mathbf{R}^{\prime}$ порождается символами

$$
\mathrm{B}(a)=\mathscr{D}_{1} A(a,-a) \mathscr{D}_{2} A(a, 0) \quad u \quad \mathrm{D} f_{i}(\vec{a})=\mathscr{D}_{1} A(f(\vec{a}),-f(\vec{a})) \mathscr{D}_{i} f(\vec{a}) \mathscr{D}_{1} A\left(0, a_{i}\right)
$$

и задается соответствующими соотношениями. В частности, для многообразия всех $\Omega$-групп $\mathbf{R}^{\prime} \cong \mathbb{Z} \mathbf{G} * \mathbf{F}_{\kappa}\left(\mathrm{D} f_{i}(\vec{a})\right)$, где первый множитель есть целочисленное групповое кольцо аддитивной группы $\mathbf{G}$, а через $\mathbf{F}_{\kappa}$ обозначено свободное кольцо.

Предлагаемая коррекция состоит в том, чтобы называть касательным кольцом не $\mathbf{R}$, а $\mathbf{R}^{\prime}$. Действительно, пусть $V$ - некоторое многообразие $\Omega$-групп, $V \ni \mathbf{G} \cong \mathbf{F}_{V}(X) / N$, где $N \triangleleft \mathbf{F}_{V}(X), N=\operatorname{ker} \pi, \pi: \mathbf{F}_{V}(X) \rightarrow \mathbf{G}$ - естественный эпиморфизм; вместо $\pi(x)$ будем также писать $\bar{x}$

Пусть $\mathbf{U}$ - свободный $\mathbf{R}$-модуль с базой $X$, и пусть множество $H=G \times U$. Тогда на $H$ вводится структура $V$-алгебры:

$$
\begin{aligned}
& (a, u)+(b, v)=\left(a+b, \mathrm{~A}(a+b)\left(\mathrm{A}(a)^{-1} u+\mathrm{B}(a) \mathrm{A}(b)^{-1} v\right)\right), \\
& \text { где } \mathrm{A}(a)=\mathscr{D}_{1}(0, a), \quad \mathrm{A}(a)^{-1}=\mathscr{D}_{1}(a,-a), \\
& -(a, u)=\left(-a,-\mathrm{A}(-a) \mathrm{B}(-a) \mathrm{A}(a)^{-1} u\right), \\
& 0=(0,0), \quad c=(c, 0), \quad \text { где } c-\text { нульарный мультиоператор, } \\
& f(\vec{h})=\left(f(\vec{a}), \mathrm{A}(f(\vec{a})) \sum_{i=1}^{n} \mathrm{D} f_{i}(\vec{a}) \mathrm{A}\left(a_{i}\right)^{-1} u_{i}\right), \\
& \text { где } h_{i}=\left(a_{i}, u_{i}\right), \quad f \text { - мультиоператор арности } n>0 .
\end{aligned}
$$

Представление Магнуса $\mu$ - это такой гомоморфизм $V$-алгебр $\mathbf{F}_{V}(X) \rightarrow \mathbf{H}$, что $\mu(x)=(\bar{x}, x)$ на $X$.

$\mathrm{C}$ другой стороны, пусть $\mathbf{U}^{\prime}$ - свободный $\mathbf{R}^{\prime}$-модуль с базой $X$, и пусть множество $H^{\prime}=$ $G \times U^{\prime}$. Тогда на $H^{\prime}$ тоже вводится структура $V$-алгебры:

$(a, u)+(b, v)=(a+b, u+\mathrm{B}(a) v), \quad-(a, u)=(-a,-\mathrm{B}(-a) u)$,

$0=(0,0), \quad c=(c, 0), \quad$ где $c-$ нульарньй мультиоператор,

$$
f(\vec{h})=\left(f(\vec{a}), \sum_{i=1}^{n} \mathrm{D} f_{i}(\vec{a}) u_{i}\right), \quad \text { где } h_{i}=\left(a_{i}, u_{i}\right), \quad f \text {-мультиоператор арности } n>0 .
$$

Обозначим через $\mu^{\prime}$ такой гомоморфизм $V$-алгебр $\mathbf{F}_{V}(X) \rightarrow \mathbf{H}^{\prime}$, что $\mu^{\prime}(x)=(\bar{x}, x)$ на $X$.

Teоpema 2. $\operatorname{ker} \mu=\operatorname{ker} \mu^{\prime}$.

На касательном кольце к любому многообразию $\Omega$-колец вводится естественная структура алгебры над основным полем. Мы будем называть ее касательной алгеброй.

Теорема 3. Касательное кольцо к многообразию всех групп в группе $\mathbf{G}$ есть $\mathbb{Z} \mathbf{G}$. Касательная алгебра к многообразию всех ассочиативных алгебр с единицей в алгебре $\mathbf{A}$ есть $\mathbf{A} \otimes \mathbf{A}^{\text {ор }}$. Касательная алгебра к многообразию всех алгебр Ли в алгебре $\mathbf{L}$ есть универсальная обертывающая для $\mathbf{L}$.

Проведенные рассмотрения существенно упрощают вычисление касательных колец к конкретным многообразиям $\Omega$-групп. В качестве примера найдено касательное кольцо к многообразию всех $p$-алгебр Ли.

\section{СПИСОК ЛИТЕРАТУРЫ}

[1] Артамонов В. А. // Сиб. матем. журн. 1997. Т. 38. № 5. С. 842-854. [2] Ремесленников В.Н., Соколов В. Г. // Алгебра и логика. 1970. Т. 9. № 5. С. 566-578. [3] Gupta N. // Contemp. Math. 1987. V. 66. P. 1-129. [4] Lewin J. // Trans. Amer. Math. Soc. 1974. V. 188. P. 293-308; 309-317. 